\title{
Existence of Mild Solutions for Multi-Term Time-Fractional Random Integro-Differential Equations with Random Carathéodory Conditions
}

\author{
Amadou Diop ${ }^{1}$ (D) and Wei-Shih Du ${ }^{2, *(D)}$ \\ 1 Department of Mathematics, Université Gaston Berger de Saint-Louis, UFR SAT, \\ Saint-Louis B.P. 234, Senegal; diop.amadou@ugb.edu.sn \\ 2 Department of Mathematics, National Kaohsiung Normal University, Kaohsiung 82444, Taiwan \\ * Correspondence: wsdu@mail.nknu.edu.tw
}

Citation: Diop, A.; Du, W.-S.

Existence of Mild Solutions for

Multi-Term Time-Fractional Random Integro-Differential Equations with Random Carathéodory Conditions. Axioms 2021, 10, 252. https:// doi.org/10.3390/axioms10040252

Academic Editors: Chris Goodrich and Davron Aslonqulovich Juraev

Received: 26 August 2021

Accepted: 1 October 2021

Published: 12 October 2021

Publisher's Note: MDPI stays neutral with regard to jurisdictional claims in published maps and institutional affiliations.

Copyright: (c) 2021 by the authors. Licensee MDPI, Basel, Switzerland. This article is an open access article distributed under the terms and conditions of the Creative Commons Attribution (CC BY) license (https:/ / creativecommons.org/licenses/by/ $4.0 /)$.

\begin{abstract}
In this paper, we investigate the existence of mild solutions to a multi-term fractional integro-differential equation with random effects. Our results are mainly relied upon stochastic analysis, Mönch's fixed point theorem combined with a random fixed point theorem with stochastic domain, measure of noncompactness and resolvent family theory. Under the condition that the nonlinear term is of Carathéodory type and satisfies some weakly compactness condition, we establish the existence of random mild solutions. A nontrivial example illustrating our main result is also given.
\end{abstract}

Keywords: measure of noncompactness; random effect; random operator; Mönch's fixed point theorem; multi-term fractional differential equation; Carathéodory condition; resolvent family theory

MSC: 34A08; 34K30; 47G20; 60H25

\section{Introduction}

For more than three decades, fractional calculus has played an important role in the study of linear and nonlinear fractional integro-differential equations that arise from the modeling of nonlinear phenomena, optimal control of complex systems and other scientific research (see, e.g., [1,2]). Multi-term time-fractional differential systems have also attracted a great interest in recent years, see for instance [3-6] and references cited therein. As inherently deterministic extensions, random fractional differential equations exist in many applications and have been studied by many authors and more details from historical points of view and recent developments of such equations are reported to the monographs $[7,8]$, papers [9-11], and the references cited therein. To be more precise, the existence results and qualitative properties for fractional differential equations with random effects are examined in $[12,13]$ and references cited therein. Very recently, considerable attention has been given to multi-term time-fractional differential systems. For instance, Pardo and Lizama [6] studied the existence of mild solutions under Carathéodory type conditions by using measure of noncompactness techniques, Singh and Pandey [14] have established the existence and uniqueness of mild solutions for multi-term time-fractional differential systems with non-instantaneous impulses and finite delay by using Banach fixed point theorem whereas Chang and Ponce [15], with the help of the theory of fractional resolvent families, established the existence of mild solutions to a multi-term fractional differential equation. It may be noted here that the mentioned works are confined to deterministic systems. Inspired by the aforementioned papers [6,12-14], this work focuses on the existence of mild solution of problem (1) with multi-term time-fractional integrodifferential equations with random effects in the form 


$$
\left\{\begin{array}{l}
{ }^{C} D^{1+\beta} \vartheta(t, \omega)+\sum_{k=1}^{n} \alpha_{k}{ }^{C} D^{\gamma_{k}} \vartheta(t, \omega)=\mathcal{A} \vartheta(t, \omega)+F\left(t, \vartheta(t, \omega), \int_{0}^{t} \mathcal{B}(t, s) \vartheta(s, \omega) d s, \omega\right), \\
\vartheta(0, \omega)=\vartheta_{0}(\omega) \\
\vartheta^{\prime}(0, \omega)=\vartheta_{1}(\omega)
\end{array}\right.
$$

for $0<t \leq b<\infty$ and $\omega \in \Omega$, where the state $\vartheta(\cdot, \cdot)$ takes values in a separable Banach space $\mathbb{X}$ with norm $\|\cdot\|,(\Omega, \mathcal{F}, \mathbb{P})$ is a complete probability space, ${ }^{C} D^{u}$ stand for the Caputo fractional derive of order $u>0,0<\beta \leq \gamma_{n} \leq \cdots \leq \gamma_{1} \leq 1$ and $\alpha_{k} \geq 0, k=1,2, \cdots n$ be given and $\mathcal{A}: \mathcal{D}(\mathcal{A}) \subset \mathbb{X} \rightarrow \mathbb{X}$ is the infinitesimal generator of a bounded and strongly continuous cosine family. $\mathrm{F}:[0, b] \times \mathbb{X} \times \mathbb{X} \times \Omega \rightarrow \mathbb{X}$ is a random nonlinear function to be specified later. The operator $\mathcal{B}(\cdot, \cdot): \Delta \rightarrow \mathbb{R}^{+}$is a continuous operator satisfying

$$
\zeta_{B}=\sup _{s, t \in[0, b]} \int_{0}^{b} \mathcal{B}(t, s) d s<\infty,
$$

where $\Delta=\left\{(t, s) \in \mathbb{R}^{2}: 0 \leq s \leq t<b\right\}$ and $\vartheta_{0}(\cdot)$ and $\vartheta_{1}(\cdot)$ are given random functions.

To the best of our knowledge, the study of the existence of mild solutions of multi-term time-fractional integro-differential equations with random effects by the abstract form (1) has not yet been treat in the literature. The main contributions of this paper are: Firstly, the study of existence of random multi-term time-fractional integro-differential equations of the form (1) via measure of noncompactness is an untreated topic in the literature. Secondly, the nonlinear term satisfies a weak compactness condition that does not require the compactness of the resolvent family and sufficient conditions for the existence of mild solutions where the solution operators are only equicontinuous, are established by means of Mönch fixed point theorem and a random fixed point theorem with stochastic domain via the noncompactness measure. At last, our theorems guarantee the effectiveness of existence results under some weakly compactness condition and the work can considered as a supplemented for the case that the corresponding $\left(\beta, \gamma_{k}\right)$-resolvent operator is compact and deterministic one. The results are established using of the $\left(\beta, \gamma_{k}\right)$-resolvent operators developed in [6].

This paper is organized as follows. Section 2 contains preliminary details. In Section 3, we show the existence of random mild solutions by Mönch's fixed point theorem combined with a fixed point theorem with stochastic domain and $\left(\beta, \gamma_{k}\right)$-resolvent family. A nontrivial example illustrating our main result (Theorem 2; see below) is also given.

\section{Preliminaries}

In this section, we recall some basic concepts, notations, definitions, lemmas, and preliminary facts, which are used throughout this article. We set $(\Omega, \mathcal{F}, P)$ be a complete probability space. Let $\mathbb{X}$ be a separable Banach space and denote $\mathcal{C}([0, b], \mathbb{X})$ be the Banach space of all continuous $\mathbb{X}$-valued functions on interval $[0, b]$ equipped with the supremum norm $\|\vartheta\|=\sup \{\|\vartheta(t)\|: t \in[0, b]\}$. In the sequel, a mapping $\vartheta:[0, b] \times \Omega \rightarrow \mathbb{X}$ is said to be a stochastic process if for each $t \in[0, b], \vartheta(t, \cdot)=\vartheta(t)(\cdot)$ is measurable. First, we recall some basics definitions and properties related to random operators which are used in this paper.

Definition 1. A mapping $\mathrm{F}: I \times \mathbb{X} \times \mathbb{X} \times \Omega \rightarrow \mathbb{X}$ is said to be random Carathéodory if the following hold:

(a) The mapping $(t, \omega) \rightarrow \mathrm{F}(t, x, y, \omega)$ is jointly measurable for all $x \in \mathbb{X}$ and for all $y \in \mathbb{X}$;

(b) The mapping $(x, y) \rightarrow \mathrm{F}(t, x, y, \omega)$ is jointly continuous for almost each $t \in[0, b]$ and for all $\omega \in \Omega$;

Definition 2 (see [16]). Let $\mathbb{X}$ be a separable Banach space with Borel $\sigma$-algebra B. A mapping $\mathrm{Y}: \Omega \times \mathbb{X} \rightarrow \mathbb{X}$ is called a random operator if $\mathrm{Y}(., y)$ is measurable for each $y \in \mathbb{X}$. 
It is generally expressed as $Y(\omega, y)=Y(\omega) y$. We will use these two expressions interchangeably in this paper.

Definition 3 (see [16]). Let $\mathrm{D}: \Omega \rightarrow 2^{\mathbb{X}}$ be a mapping and

$$
U=\{(\omega, y): \omega \in \Omega \text { and } y \in \mathrm{D}(\omega)\} .
$$

(i) A mapping $\mathrm{Y}: U \rightarrow \mathbb{X}$ is called a random operator with stochastic domain $\mathrm{D}$ if

(a) $\mathrm{D}$ is measurable (i.e, $\{\omega \in \Omega: \mathrm{D}(\omega) \cap A \neq \varnothing\} \in \mathcal{F}$ for all $A \subseteq \mathbb{X}$ );

(b) for every open set $\mathcal{O} \subseteq \mathbb{X}$ and any $y \in \mathbb{X}$,

$$
\{\omega \in \Omega: y \in \mathrm{D}(\omega) \text { and } \mathrm{Y}(\omega, y) \in \mathcal{O}\} \in \mathcal{F} .
$$

(ii) We say that $\mathrm{Y}$ is continuous if every $\mathrm{Y}(\omega)$ is continuous.

Definition 4 (see [16]). For a random operator $\mathrm{Y}$, a mapping $y: \Omega \rightarrow \mathbb{X}$ is called a random (stochastic) fixed point of $Y$ if for $\mathbb{P}$-almost all $\omega \in \Omega$, we have

$$
\begin{gathered}
y(\omega) \in \mathrm{D}(\omega), \\
\mathrm{Y}(\omega) y(\omega)=y(\omega)
\end{gathered}
$$

and

$$
\{\omega \in \Omega: y(\omega) \in \mathcal{O}\} \subset \mathcal{F}
$$

for every open set $\mathcal{O} \subseteq \mathbb{X}$ (i.e., $y$ is measurable).

Lemma 1 (see [16]). Let $(\Omega, \mathcal{F}, \mathbb{P})$ be complete and let $y_{0}: \Omega \rightarrow \mathbb{X}$ and $r: \Omega \rightarrow \mathbb{R}_{+}^{*}$ be measurable. Then $\mathrm{D}: \Omega \rightarrow 2^{\mathbb{X}}$ defined by

$$
\mathrm{D}(\omega)=\left\{y \in \mathbb{X}:\left\|y-y_{0}(\omega)\right\| \leq r(\omega)\right\}
$$

is a measurable multivalued mapping.

Lemma 2 (see [16]). Let $\mathrm{D}: \Omega \rightarrow 2^{\mathbb{X}}$ be measurable with $\mathrm{D}(\omega)$ closed, convex and solid (i.e., $\operatorname{int}(\mathrm{D}(\omega)) \neq \varnothing)$ for all $\omega \in \Omega$. Assume there exists a measurable random variable $y_{0}: \Omega \rightarrow \mathbb{X}$ with $y_{0}(\omega) \in \operatorname{int}(\mathrm{D}(\omega))$ for all $\omega \in \Omega$. Let $\mathrm{Y}$ be a continuous random operator with stochastic domain $\mathrm{D}$ such that for every $\omega \in \Omega$,

$$
\{y \in \mathrm{D}(\omega): \mathrm{Y}(\omega) y=y\} \neq \varnothing .
$$

then $\mathrm{Y}$ has a stochastic fixed point.

In this work, the existence of a mild solution to problem (1) is related to the existence of resolvent family introduce by Pardo and Lizama [6].

\subsection{Resolvent Family}

Now, we recall some definitions and basic results on fractional calculus. Let $\Gamma(\cdot)$ denote the gamma function and define $g_{x}$ for $x>0$ by

$$
g_{x}(t)=\left\{\begin{aligned}
\frac{t^{x-1}}{\Gamma(x)}, & t>0 \\
0, & t \leq 0 .
\end{aligned}\right.
$$

It is known that $g_{x}$ satisfies the following properties:

(i) for any $a, b>0,\left(g_{a} \star g_{b}\right)(t)=g_{a+b}(t)$; 
(ii) for $a, \lambda>0$ and $\operatorname{Re}(\lambda)>0, \widehat{g_{a}}(\lambda)=1 / \lambda^{a}$, where $\widehat{(\cdot)}$ and $(\cdot \star \cdot)(\cdot)$ denote the Laplace transformation and convolution, respectively.

The most frequently encountered tools in the theory of fractional calculus are provided by the Riemann-Liouville and Caputo fractional differential operators.

Definition 5. The Riemann-Liouvulle fractional integral of a function $f \in L_{l o c}^{1}([0, \infty), \mathbb{X})$ of order $\eta>0$ with lower limit zero is defined as follows

$$
\mathbb{I}^{\eta} f(t)=\left(g_{\eta} \star f\right)(t)=\int_{0}^{t} g_{\eta}(t-s) f(s) d s \quad \text { for } t>0
$$

and $\mathbb{I}^{0}(t)=f(t)$, provided that side integral is point-wise defined in $[0, \infty)$.

Definition 6. Let $\eta>0$ be given and denote $m=\lceil\eta\rceil$. The Caputo fractional derivative of order $\eta>0$ of a function $f \in C^{m}([0, \infty), \mathbb{X})$ with lower limit zero is given by

$$
{ }^{c} D^{\eta} f(t)=\mathbb{I}^{m-\eta} D^{m} f(t)=\int_{0}^{t} g_{m-\eta}(t-s) D^{m} f(s) d s,
$$

and ${ }^{c} D^{0} f(t)=f(t)$, where $D^{m}=d^{m} / d t^{m}$ and $\lceil\cdot\rceil$ is ceiling function.

For more progress and important properties about fractional calculus and its applications, we refer the reader to $[1,2]$ and references therein. The following definition was introduced by Pardo and Lizama [6] and provides a suitable representation of a mild solution for Problem (1) in terms of a specific family of bounded and linear operators.

Definition 7 (see [6]). Let $A$ be a closed linear operator on a Banach space $\mathbb{X}$ with domain $\mathcal{D}(A)$ and let $\beta>0, \gamma_{k}, \alpha_{k}, k=1,2, \cdots n$ be real positive numbers. Then $A$ is called the generator of $a\left(\beta, \gamma_{k}\right)$-resolvent family if there exists $\kappa \geq 0$ and a strongly continuous function $\mathcal{R}_{\beta, \gamma_{k}}: \mathbb{R}^{+} \rightarrow \mathcal{L}(\mathbb{X})$ such that

$$
\left\{\lambda^{\beta+1}+\sum_{k=1}^{n} \alpha_{k} \lambda^{\gamma_{k}}: \operatorname{Re}(\lambda)>\kappa\right\} \subset \rho(A)
$$

and

$$
\lambda^{\beta}\left(\lambda^{\beta+1}+\sum_{k=1}^{n} \alpha_{k} \lambda^{\gamma_{k}}-A\right)^{-1} \vartheta=\int_{0}^{\infty} e^{-\lambda t} \mathcal{R}_{\beta, \gamma_{k}}(t) \vartheta d t,
$$

where $\operatorname{Re}(\lambda)>\kappa$ and $\vartheta \in \mathbb{X}$.

Theorem 1 (see [6]). Let $0<\beta \leq \gamma_{n} \leq \gamma_{n-1} \leq \cdots \leq \gamma_{1} \leq 1$ and $\alpha_{k} \geq 0, k=1,2, \cdots, n$ be given and let $A$ be a generator of a bounded and strongly continuous cosine family $\{C(t)\}_{t \in \mathbb{R}}$. Then, A generates a bounded $\left(\beta, \gamma_{k}\right)$-resolvent family $\left(\mathcal{R}_{\beta, \gamma_{k}}(t)\right)_{t \geq 0}$.

Motivated by Pardo and Lizama [6], we introduce the concept of random mild solution for Equation (1) as follows.

Definition 8. Let $0<\beta \leq \gamma_{n} \leq \cdots \leq \gamma_{1} \leq 1$ and $\alpha_{k} \geq 0, k=1,2, \cdots n$ be given and $\mathcal{A}$ be a generator of a bounded $\left(\beta, \gamma_{k}\right)$-resolvent family $\left\{\mathcal{R}_{\beta, \gamma_{k}}(t)\right\}_{t \geq 0}$. Then, a stochastic process $\vartheta:[0, b] \times \Omega \rightarrow \mathbb{X}$ is said to be random mild solution of Equation (1) if $\vartheta(\cdot, \omega) \in \mathcal{C}([0, b], \mathbb{X})$, $\vartheta(0, \omega)=\vartheta_{0}(\omega), \vartheta^{\prime}(0, \omega)=\vartheta_{1}(\omega)$ and satifies the following integral Equation 


$$
\begin{aligned}
& \vartheta(t, \omega)=\mathcal{R}_{\beta, \gamma_{k}}(t) \vartheta_{0}(\omega)+\left(g_{1} \star \mathcal{R}_{\beta, \gamma_{k}}\right)(t) \vartheta_{1}(\omega)+\sum_{k=1}^{n} \alpha_{k} \int_{0}^{t} \frac{(t-s)^{\beta-\gamma_{k}}}{\Gamma\left(1+\beta-\gamma_{k}\right)} \mathcal{R}_{\beta, \gamma_{k}}(s) \vartheta_{0}(\omega) d s \\
&\left.+\int_{0}^{t} \mathcal{T}_{\beta, \gamma_{k}}(t-s) F\left(s, \vartheta(s, \omega), \int_{0}^{s} B(s, \tau) \vartheta(\tau, \omega) d \tau, \omega\right)\right) d s \\
& \text { for }(t, \omega) \in[0, b] \times \Omega, \text { where } \mathcal{T}_{\beta, \gamma_{k}}(t)=\left(g_{\beta} \star \mathcal{R}_{\beta, \gamma_{k}}\right)(t) .
\end{aligned}
$$

\subsection{Measures of Noncompactness}

We recall some fundamental definitions and lemmas related to the measure of noncompactness. We introduce first the definition for Hausdorff's measure of noncompactness and its properties.

Definition 9 (see [17]). The Hausdorff measure of noncompactness $\chi(\cdot)$ defined on bounded set $E$ of Banach space $\mathbb{X}$ is

$$
\chi(E)=\inf \{\epsilon>0: E \text { can be covered by finite number of balls of radii smaller then } \epsilon\} .
$$

More details on the Hausdorff's measure of noncompacness can be found in Goebel [17] and Deimling [18].

The notations $\chi(\cdot)$ and $\chi_{\mathcal{C}}(\cdot)$ stand for the Hausdorff measure of noncompactness on the bounded set of $\mathbb{X}$ and $\mathcal{C}([0, b], \mathbb{X})$, respectively. For any $V \subset \mathcal{C}([0, b], \mathbb{X})$ and $t \in[0, b]$, set $V(t)=\{\vartheta(t): \vartheta \in V\}$. Then $V(t) \subset \mathbb{X}$.

The next results play an important role in demonstrating our main result.

Lemma 3 (see [17]). Let $V \subset \mathcal{C}([0, b], \mathbb{X})$ be bounded, then

$$
\chi(V(t)) \leq \chi_{\mathcal{C}}(V) \text { for all } t \in[0, b]
$$

where $V(t)=\{v(t) ; v \in V\}$. Furthermore, if $V$ is equicontinuous on $[0, b]$, then $\chi(V(t))$ is continuous on $[0, b]$, and $\chi_{\mathcal{C}}(V)=\sup _{t \in[0, b]} \alpha(V(t))$.

Lemma 4 (see [19]). Let $\left\{Z_{n}: n \in \mathbb{N}\right\}$ be a sequence of Bochner integrable functions from $[0, b]$ into $\mathbb{X}$ such that $\left\|Z_{n}(t)\right\| \leq f(t)$ for every $n \geq 1$ and almost all $t \in[0, b]$, where $f \in$ $\mathbb{L}^{1}\left([0, b], \mathbb{R}^{+}\right)$, then the function $\bar{Z}(t)=\chi\left\{Z_{n}(t): n \geq 1\right\} \in \mathbb{L}^{1}\left([0, b], \mathbb{R}^{+}\right)$and satisfy

$$
\chi\left(\int_{0}^{t} Z_{n}(s) d s: n \geq 1\right) \leq 2 \int_{0}^{t} Z(s) d s .
$$

Definition 10 (see [20]). A countable set $\left\{Z_{n}: n \geq 1\right\} \subset \mathbb{L}^{1}([0, b], \mathbb{X})$ is called semicompact if there exists a function $f \in \mathbb{L}^{1}\left([0, b], \mathbb{R}^{+}\right)$satisfying $\sup _{n \geq 1} Z_{n}(t) \leq f(t)$ for a.e. $t \in[0, b]$ and the sequence $\left\{Z_{n}: n \geq 1\right\}$ is relatively compact in $\mathbb{X}$.

Lemma 5. [20] Let $(\mathbb{Q} Z)(t)=\int_{0}^{t} \mathcal{T}_{\beta, \gamma_{k}}(t-s) Z(s) d s$ and the sequence $\left\{Z_{n}: n \geq 1\right\} \subset$ $\mathbb{L}^{1}([0, b], \mathbb{X})$ be semicompact. Then the following statements hold:

(i) The set $\left\{\mathbb{Q} Z_{n}: n \geq 1\right\} \subset \mathbb{L}^{1}([0, b], \mathbb{X})$ is relatively compact in $\mathcal{C}([0, b], \mathbb{X})$.

(ii) If $Z_{n} \rightarrow Z$, then $\left(\mathbb{Q} Z_{n}\right)(t) \rightarrow(\mathbb{Q} Z)(t)$, as $n \rightarrow \infty$, for all $t \in[0, b]$.

To prove our existence results, we shall use the following Mönch's fixed point theorem combined with the stochastic fixed point theorem (i.e., Lemma 2). 
Lemma 6 (see [21]). Let $\mathbb{V}$ be a closed and convex subset of $\mathbb{X}$ and $0 \in \mathbb{V}$. Then a continuous mapping $\mathcal{Q}: \mathbb{V} \rightarrow \mathbb{X}$ which satisies Mönch condition (i.e., $\mathbb{W} \subseteq \mathbb{V}$ is countable and $\mathbb{W} \subseteq$ $\overline{\operatorname{con}}(\{0\} \cup \mathcal{Q}(\mathbb{W})) \Longrightarrow \overline{\mathbb{W}}$ is compact) has a fixed point in $\mathbb{V}$.

\section{Some Existence Results}

In this section, we investigate the existence of random mild solution for Equation (1). The following conditions will be used in our main theorem.

(H1) $\mathcal{A}$ be a generator of a bounded $\left(\beta, \gamma_{k}\right)$-resolvent family $\left\{\mathcal{R}_{\beta, \gamma_{k}}(t)\right\}_{t \geq 0}$ which is equicontinous. Let $M=\sup _{t \in[0, b]}\left\|\mathcal{R}_{\beta, \gamma_{k}}(t)\right\|_{\mathcal{L}(\mathbb{X})}$.

(H2) Then function $F:[0, b] \times \mathbb{X} \times \mathbb{X} \times \Omega \rightarrow \mathbb{X}$ is random Caratheodory and the functions $\vartheta_{0}(\cdot): \Omega \rightarrow \mathbb{X}$ and $\vartheta_{1}(\cdot): \Omega \rightarrow \mathbb{X}$ are measurables and essentialy bounded. Let

$$
\max \left\{\operatorname{ess} \sup _{\omega \in \Omega}\left\|\vartheta_{0}(\omega)\right\| ; \text { ess } \sup _{\omega \in \Omega}\left\|\vartheta_{1}(\omega)\right\|\right\} \leq c_{0}
$$

for some constant $c_{0} \in \mathbb{R}^{+}$.

(H3) There exist two functions $f:[0, b] \times \Omega \rightarrow \mathbb{R}^{+}$and $G: \mathbb{R}^{+} \times \Omega \rightarrow \mathbb{R}^{+}$such that for each $\omega \in \Omega, f(\cdot, \omega) \in L^{1 / p_{1}}\left([0, b], \mathbb{R}^{+}\right)$for a constant $p_{1} \in(0,1)$ and $G(\cdot, \omega)$ a nondeacresing continuous function with

$\|F(t, x, y, \omega)\|_{\mathbb{X}} \leq f(t, \omega) G(\|x\|+\|y\|, \omega)$, for a.e $t \in[0, b]$ and each $x \in \mathbb{X}, y \in \mathbb{X}$.

(H4) For a constant $p_{2} \in(0,1)$ and bounded subsets $\mathbb{V}_{1}, \mathbb{V}_{2} \subset \mathbb{X}$, there exists a function $h:[0, b] \times \Omega \rightarrow \mathbb{R}^{+}$such that for each $\omega \in \Omega, h(\cdot, \omega) \in \mathbb{L}^{1 / p_{2}}\left([0, b], \mathbb{R}^{+}\right)$with

$$
\chi\left(F\left(t, \mathbb{V}_{1}, \mathbb{V}_{2}, \omega\right)\right) \leq h(t, \omega)\left[\chi\left(\mathbb{V}_{1}\right)+\chi\left(\mathbb{V}_{2}\right)\right]
$$

(H5) There exists a random function $r: \Omega \rightarrow \mathbb{R} \backslash\{0\}$ such that

$$
\begin{aligned}
& M c_{0}\left[1+b+\sum_{k=1}^{n} \frac{\alpha_{k} b^{\left(1+\beta-\gamma_{k}\right)}}{\Gamma\left(2+\beta-\gamma_{k}\right)}\right]+\frac{M G\left(\left(1+\zeta_{B}\right) r(\omega), \omega\right)}{\Gamma(1+\beta)} \widehat{P}_{1}\|f(\cdot, \omega)\|_{\mathbb{L}^{1 / p_{1}}} \leq r(\omega) \\
& \text { where } \widehat{P}_{1}=\left[\left(\frac{1-p_{1}}{1+\beta-p_{1}}\right) b^{\left(1+\beta-p_{1}\right) /\left(1-p_{1}\right)}\right]^{1-p_{1}} .
\end{aligned}
$$

The following existence theorem is one of the main results of this paper.

Theorem 2. Assume that the assumptions (H1)-(H5) are valid, then the multi-term time-fractional integro-differential problem (1) has at least one mild random solution on $[0, b]$ provided that

$$
\frac{M \widehat{P}_{2}\left(1+\zeta_{B}\right)}{\Gamma(1+\beta)}\|h(\cdot, \omega)\|_{\mathbb{L}^{1 / p_{2}}}<\frac{1}{2}
$$

where $\widehat{P}_{2}=\left[\left(\frac{1-p_{2}}{1+\beta-p_{2}}\right) b^{\left(1+\beta-p_{2}\right) /\left(1-p_{2}\right)}\right]^{1-p_{2}}$.

Proof. It is noted that

$$
\left\|\mathcal{T}_{\beta, \gamma_{k}}(t)\right\|_{\mathcal{L}(\mathbb{X})} \leq \frac{M t^{\beta}}{\Gamma(1+\beta)}
$$


Consider the random operator $\mathcal{N}: \Omega \times \mathcal{C}([0, b], \mathbb{X}) \rightarrow \mathcal{C}([0, b], \mathbb{X})$ defined by

$$
\begin{aligned}
(\mathcal{N}(\omega) \vartheta)(t) & =\mathcal{R}_{\beta, \gamma_{k}}(t) \vartheta_{0}(\omega)+\left(g_{1} \star \mathcal{R}_{\beta, \gamma_{k}}\right)(t) \vartheta_{1}(\omega) \\
& +\sum_{k=1}^{n} \alpha_{k} \int_{0}^{t} \frac{(t-s)^{\beta-\gamma_{k}}}{\Gamma\left(1+\beta-\gamma_{k}\right)} \mathcal{R}_{\beta, \gamma_{k}}(s) \vartheta_{0}(\omega) d s \\
& \left.+\int_{0}^{t} \mathcal{T}_{\beta, \gamma_{k}}(t-s) F\left(s, \vartheta(s, \omega), \int_{0}^{s} \mathcal{B}(s, \tau) \vartheta(\tau, \omega) d \tau, \omega\right)\right) d s
\end{aligned}
$$

for $t \in[0, b]$. We divide the proof into a sequence of steps.

Step 1. We show that the mapping $\mathcal{N}$ is a random operator with stochastic domain. By assumption (H2), we know that $F(t, x, y, \cdot)$ for $t \in[0, b], x, y \in \mathbb{X}, \vartheta_{0}(\cdot)$ and $\vartheta_{1}(\cdot)$ are measurables. Then $\mathcal{N}(\cdot) \vartheta: \Omega \rightarrow \mathcal{C}([0, b], \mathbb{X})$ is a random varriable. Let $D: \Omega \rightarrow 2^{\mathcal{C}([0, b], \mathbb{X})}$ be defined by

$$
D(\omega)=\{\vartheta \in \mathcal{C}([0, b], \mathbb{X}):\|\vartheta\| \leq r(\omega)\} .
$$

Thus, the set $D(\omega)$ is bounded, closed, convex and solid for all $\omega \in \Omega$. So $D$ is measurable by Lemma 1. For each $\vartheta \in D(\omega)$, using (H2), (H3), (H4), and Hölder's inequality, we have

$$
\begin{aligned}
& \|(\mathcal{N}(\omega) \vartheta)(t)\| \\
\leq & \left\|\mathcal{R}_{\beta, \gamma_{k}}(t) \vartheta_{0}(\omega)\right\|+\left\|\left(g_{1} \star \mathcal{R}_{\beta, \gamma_{k}}\right)(t) \vartheta_{1}(\omega)\right\|+\left\|\sum_{k=1}^{n} \alpha_{k} \int_{0}^{t} \frac{(t-s)^{\beta-\gamma_{k}}}{\Gamma\left(1+\beta-\gamma_{k}\right)} \mathcal{R}_{\beta, \gamma_{k}}(s) \vartheta_{0}(\omega) d s\right\| \\
& +\left\|\int_{0}^{t} \mathcal{T}_{\beta, \gamma_{k}}(t-s) F\left(s, \vartheta(s, \omega), \int_{0}^{s} B(s, \tau) \vartheta(\tau, \omega) d \tau, \omega\right) d s\right\| \\
\leq & M c_{0}+b M c_{0}+\sum_{k=1}^{n} \frac{\alpha_{k} M c_{0} b^{\left(1+\beta-\gamma_{k}\right)}}{\Gamma\left(2+\beta-\gamma_{k}\right)} \\
& +\int_{0}^{t}\left\|\mathcal{T}_{\beta, \gamma_{k}}(t-s)\right\|\left\|_{\mathcal{L}(\mathbb{X})}\right\| F\left(s, \vartheta(s, \omega), \int_{0}^{s} \mathcal{B}(s, \tau) \vartheta(\tau, \omega) d \tau, \omega\right) \| d s \\
\leq & M c_{0}+b M c_{0}+\sum_{k=1}^{n} \frac{\alpha_{k} M c_{0} b^{\left(1+\beta-\gamma_{k}\right)}}{\Gamma\left(2+\beta-\gamma_{k}\right)} \\
& +\frac{M}{\Gamma(1+\beta)} \int_{0}^{t}(t-s)^{\beta} f(s, \omega) G\left(\|\vartheta(t, \omega)\|+\left\|\int_{0}^{s} \mathcal{B}(s, \tau) \vartheta(\tau, \omega) d \tau\right\|, \omega\right) \\
\leq & M c_{0}+b M c_{0}+\sum_{k=1}^{n} \frac{\alpha_{k} M c_{0} b^{\left(1+\beta-\gamma_{k}\right)}}{\Gamma\left(2+\beta-\gamma_{k}\right)}+\frac{M}{\Gamma(1+\beta)} \int_{0}^{b}(b-s)^{\beta} f(s, \omega) G\left(\left(1+\zeta_{B}\right) r(\omega), \omega\right) d s \\
\leq & M c_{0}+b M c_{0}+\sum_{k=1}^{n} \frac{\alpha_{k} M c_{0} b^{\left(1+\beta-\gamma_{k}\right)}}{\Gamma\left(2+\beta-\gamma_{k}\right)} \\
& +\frac{M G\left(\left(1+\zeta_{B}\right) r(\omega), \omega\right)}{\Gamma(1+\beta)}\left(\int_{0}^{b}(b-s)^{\beta /\left(1-p_{1}\right)} d s\right)^{1-p_{1}}\left(\int_{0}^{b}(f(s, \omega))^{1 / p_{1}} d s\right)^{p_{1}}
\end{aligned}
$$




$$
\begin{aligned}
\leq & M c_{0}+b M c_{0}+\sum_{k=1}^{n} \frac{\alpha_{k} M c_{0} b^{\left(1+\beta-\gamma_{k}\right)}}{\Gamma\left(2+\beta-\gamma_{k}\right)} \\
& +\frac{M G\left(\left(1+\zeta_{B}\right) r(\omega), \omega\right)}{\Gamma(1+\beta)}\left[\left(\frac{1-p_{1}}{1+\beta-p_{1}}\right) b^{\left(1+\beta-p_{1}\right) /\left(1-p_{1}\right)}\right]^{1-p_{1}}\|f(\cdot, \omega)\|_{\mathbb{L}^{1 / p_{1}}} \\
\leq & M c_{0}\left[1+b+\sum_{k=1}^{n} \frac{\alpha_{k} b^{\left(1+\beta-\gamma_{k}\right)}}{\Gamma\left(2+\beta-\gamma_{k}\right)}\right]+\frac{M G\left(\left(1+\zeta_{B}\right) r(\omega), \omega\right)}{\Gamma(1+\beta)} \widehat{P}_{1}\|f(\cdot, \omega)\|_{\mathbb{L}^{1 / p_{1}}} \\
\leq & r(\omega),
\end{aligned}
$$

which implies that $\mathcal{N}$ is a random operator with stochastic domain $D$ and $\mathcal{N}(\omega): D(\omega) \rightarrow$ $D(\omega)$ for each $\omega \in \Omega$.

Step 2. Show that $\mathcal{N}$ is continuous on $D(\omega)$.

Let $\left(\vartheta^{(m)}\right)_{m \in \in \mathbb{N}}$ be a sequence in $D(\omega)$ satisfying $\vartheta^{m} \rightarrow \vartheta$ in $D(\omega)$ and define

$$
\widetilde{F}_{m}(s)=F\left(s, \vartheta^{(m)}(s, \omega), \int_{0}^{s} \mathcal{B}(s, \tau) \vartheta^{(m)}(\tau, \omega) d \tau, \omega\right)
$$

and

$$
\widetilde{F}(s)=F\left(s, \vartheta(s, \omega), \int_{0}^{s} \mathcal{B}(s, \tau) \vartheta(\tau, \omega) d \tau, \omega\right)
$$

then

$$
\left\|\left(\mathcal{N}(\omega) \vartheta^{(m)}\right)(t)-(\mathcal{N}(\omega) \vartheta)(t)\right\| \leq \frac{M}{\Gamma(1+\beta)} \int_{0}^{t}(t-s)^{\beta}\left\|\widetilde{F}_{m}(s)-\widetilde{F}(s)\right\| d s .
$$

by assumption (H2) and combining with Lebesgue dominated convergence theorem, we get

$$
\int_{0}^{t}(t-s)^{\beta}\left\|\widetilde{F}_{m}(s)-\widetilde{F}(s)\right\| d s \rightarrow 0 \text { as } m \rightarrow \infty, \text { for } t \in[0, b] .
$$

consequently, we obtain

$$
\left\|\left(\mathcal{N}(\omega) \vartheta^{(m)}\right)(t)-(\mathcal{N}(\omega) \vartheta)(t)\right\| \rightarrow 0 \text { as } m \rightarrow \infty .
$$

hence $\mathcal{N}$ is continuous.

Step 3. Show that for every $\omega \in \Omega,\{\vartheta \in D(\omega): \mathcal{N}(\omega) \vartheta=\vartheta\} \neq \varnothing$.

To achieve this, we going to demontrate that the Mönch condition holds. Let $\omega \in \Omega$ be arbitrary fixed. First, let show that $\mathcal{N}$ maps bounded sets into equicontinuous sets of $D(\omega)$. Let $t_{1}, t_{2} \in[0, b]$ with $t_{2}>t_{1}$ and $\vartheta \in D(\omega)$. By the equicontinuouty of $\mathcal{R}_{\beta, \gamma_{k}}(t)$, we have 


$$
\begin{aligned}
& \left\|(\mathcal{N}(\omega) \vartheta)\left(t_{2}\right)-(\mathcal{N}(\omega) \vartheta)\left(t_{1}\right)\right\| \\
& \leq\left\|\mathcal{R}_{\beta, \gamma_{k}}\left(t_{2}\right)-\mathcal{R}_{\beta, \gamma_{k}}\left(t_{1}\right)\right\|_{\mathcal{L}(\mathbb{X})}\left\|\vartheta_{0}(\omega)\right\| \\
& +\left\|\left(g_{1} \star \mathcal{R}_{\beta, \gamma_{k}}\right)\left(t_{2}\right) \vartheta_{1}(\omega)-\left(g_{1} \star \mathcal{R}_{\beta, \gamma_{k}}\right)\left(t_{1}\right) \vartheta_{1}(\omega)\right\| \\
& +\left\|\sum_{k=1}^{n} \alpha_{k} \int_{0}^{t_{2}} \frac{\left(t_{2}-s\right)^{\beta-\gamma_{k}}}{\Gamma\left(1+\beta-\gamma_{k}\right)} \mathcal{R}_{\beta, \gamma_{k}}(s) \vartheta_{0}(\omega) d s-\sum_{k=1}^{n} \alpha_{k} \int_{0}^{t_{1}} \frac{\left(t_{1}-s\right)^{\beta-\gamma_{k}}}{\Gamma\left(1+\beta-\gamma_{k}\right)} \mathcal{R}_{\beta, \gamma_{k}}(s) \vartheta_{0}(\omega) d s\right\| \\
& +\left\|\int_{0}^{t_{2}} \mathcal{T}_{\beta, \gamma_{k}}\left(t_{2}-s\right) \widetilde{F}(s) d s-\int_{0}^{t_{1}} \mathcal{T}_{\beta, \gamma_{k}}\left(t_{1}-s\right) \widetilde{F}(s) d s\right\| \\
& \leq\left\|\mathcal{R}_{\beta, \gamma_{k}}\left(t_{2}\right)-\mathcal{R}_{\beta, \gamma_{k}}\left(t_{1}\right)\right\|_{\mathcal{L}(\mathbb{X}}\left\|\vartheta_{0}(\omega)\right\| \\
& +\int_{t_{1}}^{t_{2}}\left\|\mathcal{R}_{\beta, \gamma_{k}}(s)\right\|_{\mathcal{L}(\mathbb{X}}\left\|\vartheta_{1}(\omega)\right\| d s \\
& +\sum_{k=1}^{n} \alpha_{k}\left\|\int_{0}^{t_{2}} \frac{\left(t_{2}-s\right)^{\beta-\gamma_{k}}}{\Gamma\left(1+\beta-\gamma_{k}\right)} \mathcal{R}_{\beta, \gamma_{k}}(s) d s-\int_{0}^{t_{1}} \frac{\left(t_{1}-s\right)^{\beta-\gamma_{k}}}{\Gamma\left(1+\beta-\gamma_{k}\right)} \mathcal{R}_{\beta, \gamma_{k}}(s) d s\right\|\left\|\vartheta_{0}(\omega)\right\| \\
& +\int_{0}^{t_{1}}\left\|\mathcal{T}_{\beta, \gamma_{k}}\left(t_{2}-s\right)-\mathcal{T}_{\beta, \gamma_{k}}\left(t_{1}-s\right)\right\|\|\widetilde{F}(s)\| d s-\int_{t_{1}}^{t_{2}}\left\|\mathcal{T}_{\beta, \gamma_{k}}\left(t_{1}-s\right)\right\|\|\widetilde{F}(s)\| d s \\
& \leq c_{0}\left\|\mathcal{R}_{\beta, \gamma_{k}}\left(t_{2}\right)-\mathcal{R}_{\beta, \gamma_{k}}\left(t_{1}\right)\right\|_{\mathcal{L}(\mathbb{X})}+M c_{0}\left(t_{2}-t_{1}\right) \\
& +\sum_{k=1}^{n} \frac{\alpha_{k} M}{\Gamma\left(1+\beta-\gamma_{k}\right)}\left[\int_{0}^{t_{1}}\left|\left(t_{2}-s\right)^{\beta-\gamma_{k}}-\left(t_{1}-s\right)^{\beta-\gamma_{k}}\right| d s-\int_{t_{1}}^{t_{2}}\left|\left(t_{2}-s\right)\right|^{\beta-\gamma_{k}} d s\right] c_{0} \\
& +\int_{0}^{t_{1}}\left\|\mathcal{T}_{\beta, \gamma_{k}}\left(t_{2}-s\right)-\mathcal{T}_{\beta, \gamma_{k}}\left(t_{1}-s\right)\right\|\|\widetilde{F}(s)\| d s+\frac{M}{\Gamma(1+\beta)} \int_{t_{1}}^{t_{2}}\left|\left(t_{1}-s\right)\right|^{\beta}\|\widetilde{F}(s)\| d s \\
& \leq c_{0}\left\|\mathcal{R}_{\beta, \gamma_{k}}\left(t_{2}\right)-\mathcal{R}_{\beta, \gamma_{k}}\left(t_{1}\right)\right\|_{\mathcal{L}(\mathbb{X})}+M c_{0}\left(t_{2}-t_{1}\right) \\
& +\sum_{k=1}^{n} \frac{\alpha_{k} M}{\Gamma\left(1+\beta-\gamma_{k}\right)}\left[\int_{0}^{t_{1}}\left|\left(t_{2}-s\right)^{\beta-\gamma_{k}}-\left(t_{1}-s\right)^{\beta-\gamma_{k}}\right| d s-\int_{t_{1}}^{t_{2}}\left|\left(t_{2}-s\right)\right|^{\beta-\gamma_{k}} d s\right] c_{0} \\
& +\int_{0}^{t_{1}}\left\|\mathcal{T}_{\beta, \gamma_{k}}\left(t_{2}-t_{1}+s\right)-\mathcal{T}_{\beta, \gamma_{k}}(s)\right\|\|\widetilde{F}(s)\| d s+\frac{M}{\Gamma(1+\beta)} \int_{t_{1}}^{t_{2}}\left|\left(t_{1}-s\right)\right|^{\beta}\|\widetilde{F}(s)\| d s .
\end{aligned}
$$

By the equicontinuity of $\mathcal{R}_{\beta, \gamma_{k}}$ and Lebesgue dominated convergence theorem, we conclude that the right side of the above inequality tends to zero (independently of $\vartheta$ ) as $t_{1} \rightarrow t_{2}$. Thus, $\mathcal{N}(\omega)(\mathbb{V})$ is equicontinuous on $[0, b]$.

Now, let us assume that $\mathbb{V}=\left\{\vartheta^{(m)}\right\}_{m=1}^{\infty}$ be a countable subset of $D(\omega)$ and $\mathbb{V} \subseteq$ $\overline{\operatorname{con}}(\{0\} \cup \mathcal{N}(\omega)(\mathbb{V}))$. Since $\mathcal{N}(\omega)(\mathbb{V})$ is is bounded and equicontinuous, we have $\mathbb{V}=$ $\left\{\vartheta^{(m)}\right\}_{m=1}^{\infty}$ is bounded and equicontinuous and therefore by Lemma 3 , the function $t \rightarrow$ $\chi(\mathbb{V}(t))$ is continuous on $[0, b]$. By Lemma 4 , we get 


$$
\begin{aligned}
& \chi\left(\left\{\left(\mathcal{N}(\omega) \vartheta^{(m)}\right)(t)\right\}_{m=1}^{\infty}\right) \\
\leq & \chi\left(\left\{\int_{0}^{t} \mathcal{T}_{\beta, \gamma_{k}}(t-s) F\left(s, \vartheta^{(m)}(s, \omega), \int_{0}^{s} \mathcal{B}(s, \tau) \vartheta^{(m)}(\tau, \omega) d \tau, \omega\right) d s\right\}_{m=1}^{\infty}\right) \\
\leq & 2 \frac{M\left(1+\zeta_{B}\right)}{\Gamma(1+\beta)} \int_{0}^{t}(t-s)^{\beta} h(s, \omega) \chi\left(\left\{\vartheta^{(m)}(s)\right\}_{m=1}^{\infty}\right) d s \\
\leq & 2 \frac{M\left(1+\zeta_{B}\right)}{\Gamma(1+\beta)} \int_{0}^{b}(b-s)^{\beta} h(s, \omega) \sup _{s \in[0, b]} \chi\left(\left\{\vartheta^{(m)}(s)\right\}_{m=1}^{\infty}\right) d s \\
\leq & 2 \frac{M\left(1+\zeta_{B}\right)}{\Gamma(1+\beta)} \sup _{s \in[0, b]} \chi\left(\left\{\vartheta^{(m)}(s)\right\}_{m=1}^{\infty}\right)\left(\int_{0}^{b}(b-s)^{\beta /\left(1-p_{2}\right)} d s\right)^{1-p_{2}}\left(\int_{0}^{b}(h(s, \omega))^{1 / p_{2}} d s\right)^{p_{2}} \\
\leq & 2 \frac{M\left(1+\zeta_{B}\right)}{\Gamma(1+\beta)} \sup _{s \in[0, b]} \chi\left(\left\{\vartheta^{(m)}(s)\right\}_{m=1}^{\infty}\right) K_{2}\|h(\cdot, \omega)\|_{\mathbb{L}^{1 / p_{2}}} .
\end{aligned}
$$

By Lemma 3, we obtain

$$
\chi(\mathcal{N}(\omega) \mathbb{V}) \leq\left[2 \frac{M K_{2}\left(1+\zeta_{B}\right)}{\Gamma(1+\beta)}\|h(\cdot, \omega)\|_{\mathbb{L}^{1 / p_{2}}}\right] \chi(\mathbb{V}) .
$$

From Mönch condition, we get

$$
\chi(\mathbb{V}) \leq \chi(\overline{\operatorname{con}}(\{0\} \cup \mathcal{N}(\omega) \mathbb{V}))=\chi(\mathcal{N}(\omega) \mathbb{V}) \leq\left[2 \frac{M K_{2}\left(1+\zeta_{B}\right)}{\Gamma(1+\beta)}\|h(\cdot, \omega)\|_{\mathbb{L}^{1 / p_{2}}}\right] \chi(\mathbb{V})
$$

From inequality (2), we deduce that $\chi(\mathbb{V})=0$. As a consequence of Theorem 6 , we show that $\mathcal{N}$ has a fixed point $\vartheta(\omega) \in D(\omega)$. Since $\cap_{\omega \in \Omega} D(\omega) \neq \varnothing$, the hypothesis that a measurable selector of $\operatorname{int}(D)$ holds. By Lemma 2, the random operator $\mathcal{N}$ has a stochastic fixed point $\vartheta^{\star}(\omega)$, which is a mild solution of (1). This complete the proof.

With another growth condition of $F(\cdot, \cdot, \cdot)$, the condition (H4) can be deleted. Precisely, we replace the assumption $(\mathbf{H} 3)$ with $\left(\mathbf{H} \mathbf{3}^{\prime}\right)$, where

$\left(\mathbf{H 3}^{\prime}\right)$ There exist two functions $Z_{1}, Z_{2}:[0, b] \times \Omega \rightarrow \mathbb{R}^{+}$such that for each $\omega \in \Omega$, $Z_{1}(\cdot, \omega), Z_{2}(\cdot, \omega) \in L^{1 / p_{1}}\left([0, b], \mathbb{R}^{+}\right)$for a constant $p_{1} \in(0,1)$ with

$$
\|F(t, x, y, \omega)\|_{\mathbb{X}} \leq Z_{1}(t, \omega)+Z_{2}(t, \omega)(\|x\|+\|y\|)
$$

for $t \in[0, b]$ and $x, y \in \mathbb{X}$.

Theorem 3. Under the assumptions (H1), (H2), (H3'), (H4) and condition (2), the multi-term time-fractional integro-differential problem (1) has at least one mild random solution on $[0, b]$.

Proof. Using (H1), (H2), (H3' $\left.{ }^{\prime}\right)$, and Hölder's inequality, we have 
$\|(\mathcal{N}(\omega) \vartheta)(t)\|$

$\leq\left\|\mathcal{R}_{\beta, \gamma_{k}}(t) \vartheta_{0}(\omega)\right\|+\left\|\left(g_{1} \star \mathcal{R}_{\beta, \gamma_{k}}\right)(t) \vartheta_{1}(\omega)\right\|+\left\|\sum_{k=1}^{n} \alpha_{k} \int_{0}^{t} \frac{(t-s)^{\beta-\gamma_{k}}}{\Gamma\left(1+\beta-\gamma_{k}\right)} \mathcal{R}_{\beta, \gamma_{k}}(s) \vartheta_{0}(\omega) d s\right\|$

$+\left\|\int_{0}^{t} \mathcal{T}_{\beta, \gamma_{k}}(t-s) F\left(s, \vartheta(s, \omega), \int_{0}^{s} B(s, \tau) y(\tau, \omega) d \tau, \omega\right) d s\right\|$

$\leq M c_{0}+b M c_{0}+\sum_{k=1}^{n} \frac{\alpha_{k} M c_{0} b^{\left(1+\beta-\gamma_{k}\right)}}{\Gamma\left(2+\beta-\gamma_{k}\right)}$

$+\int_{0}^{t}\left\|\mathcal{T}_{\beta, \gamma_{k}}(t-s)\right\|_{\mathcal{L}(\mathbb{X})}\left\|F\left(s, \vartheta(s, \omega), \int_{0}^{s} \mathcal{B}(s, \tau) \vartheta(\tau, \omega) d \tau, \omega\right)\right\| d s$

$\leq M c_{0}+b M c_{0}+\sum_{k=1}^{n} \frac{\alpha_{k} M c_{0} b^{\left(1+\beta-\gamma_{k}\right)}}{\Gamma\left(2+\beta-\gamma_{k}\right)}$

$\left.+\frac{M}{\Gamma(1+\beta)} \int_{0}^{t}(t-s)^{\beta}\left[Z_{1}(s, \omega)+Z_{2}(s, \omega)\left(\|\vartheta(t, \omega)\|+\left\|\int_{0}^{s} \mathcal{B}(s, \tau) \vartheta(\tau, \omega) d \tau\right\|\right)\right)\right] d s$

$\leq M c_{0}+b M c_{0}+\sum_{k=1}^{n} \frac{\alpha_{k} M c_{0} b^{\left(1+\beta-\gamma_{k}\right)}}{\Gamma\left(2+\beta-\gamma_{k}\right)}$

$+\frac{M}{\Gamma(1+\beta)} \int_{0}^{b}(b-s)^{\beta}\left[Z_{1}(s, \omega)+Z_{2}(s, \omega)\left(1+\zeta_{B}\right)\|\vartheta\|\right] d s$

$\leq M c_{0}+b M c_{0}+\sum_{k=1}^{n} \frac{\alpha_{k} M c_{0} b^{\left(1+\beta-\gamma_{k}\right)}}{\Gamma\left(2+\beta-\gamma_{k}\right)}+\frac{M}{\Gamma(1+\beta)}\left(\int_{0}^{b}(b-s)^{\beta /\left(1-p_{1}\right)} d s\right)^{1-p_{1}}\left(\int_{0}^{b}\left(Z_{1}(s, \omega)\right)^{1 / p_{1}} d s\right)^{p_{1}}$

$+\frac{M\left(1+\zeta_{B}\right)}{\Gamma(1+\beta)}\|\vartheta\|\left(\int_{0}^{b}(b-s)^{\beta /\left(1-p_{1}\right)} d s\right)^{1-p_{1}}\left(\int_{0}^{b}\left(Z_{2}(s, \omega)\right)^{1 / p_{1}} d s\right)^{p_{1}}$

$\leq M\left[c_{0}+b c_{0}+\sum_{k=1}^{n} \frac{\alpha_{k} c_{0} b^{\left(1+\beta-\gamma_{k}\right)}}{\Gamma\left(2+\beta-\gamma_{k}\right)}+\frac{1}{\Gamma(1+\beta)} \widehat{P}_{1}\left(\int_{0}^{b}\left(Z_{1}(s, \omega)\right)^{1 / p_{1}} d s\right)^{p_{1}}\right]$

$+M \frac{\left(1+\zeta_{B}\right)}{\Gamma(1+\beta)} \widehat{P}_{1}\left(\int_{0}^{b}\left(Z_{2}(s, \omega)\right)^{1 / p_{1}} d s\right)^{p_{1}}\|\vartheta\|$

$\leq M K_{1}(\omega)+M K_{2}(\omega)\|\vartheta\|$,

where

$$
K_{1}(\omega)=c_{0}+b c_{0}+\sum_{k=1}^{n} \frac{\alpha_{k} c_{0} b^{\left(1+\beta-\gamma_{k}\right)}}{\Gamma\left(2+\beta-\gamma_{k}\right)}+\frac{1}{\Gamma(1+\beta)} \widehat{P}_{1}\left(\int_{0}^{b}\left(Z_{1}(s, \omega)\right)^{1 / p_{1}} d s\right)^{p_{1}}
$$

and

$$
K_{2}(\omega)=M \frac{\left(1+\zeta_{B}\right)}{\Gamma(1+\beta)} \widehat{P}_{1}\left(\int_{0}^{b}\left(Z_{2}(s, \omega)\right)^{1 / p_{1}} d s\right)^{p_{1}} .
$$




$$
\begin{aligned}
\text { choosing } r(\omega)= & \frac{M K_{1}(\omega)}{1-M K_{2}(\omega)} \text { and } \\
& D(\omega)=\{\vartheta \in \mathcal{C}([0, b], \mathbb{X}):\|\vartheta\| \leq r(\omega)\} \text { for all } \vartheta \in D(\omega),
\end{aligned}
$$

we get

$$
\|(\mathcal{N}(\omega) \vartheta))(t) \| \leq r(\omega) .
$$

The proof can be complete similarly to Theorem 2 .

Remark 1. Consider the measure of noncompactness $\chi_{\mathcal{C}}$ and $v$ defined in $\mathcal{C}([0, b], \mathbb{X})$ by

$$
\chi_{\mathcal{C}}(\mathbb{K})=\max _{\mathbb{O} \in \Delta(\mathbb{K})}\left\{v(\mathbb{O}), \bmod _{\mathcal{c}}(\mathbb{O})\right\} \quad \text { and } \quad v(\mathbb{O})=\sup _{t \in[0, b]} e^{-L t} \chi(\mathbb{O}(t)),
$$

for all bounded subsets $\mathbb{K}$ of $\mathcal{C}([0, b], \mathbb{X})$, where $\Delta(\mathbb{K})$ stand for the collection of all countable subsets of $\mathbb{K}, \mathbb{O}(t)=\{x(t), x \in \mathbb{O}, t \in[0, b]\}$ and $L$ is an appropriate constant to be defined later. mod $_{C}$ is the modulus of equicontinuity of the function set $\mathbb{O}$ defined by

$$
\bmod _{C}(\mathbb{O})=\lim _{\delta \rightarrow 0} \sup _{x \in \mathbb{O}} \max \left\{\left\|x\left(t_{2}\right)-x\left(t_{1}\right)\right\|: t_{1}, t_{2} \in[0, b],\left|t_{2}-t_{1}\right| \leq \delta\right\} .
$$

From [20], we know that there exists a $\mathbb{O}^{\star}$ which achieves the maximun in (5). Furthermore, the measure $\chi_{\mathcal{C}}$ is nonsingular, monotone and regular.

Applying the abaove regular measure of noncompactness, we obtain the following result.

Theorem 4. Assume that the assumptions $(\mathrm{H1})-(\mathrm{H} 5)$ are valid, then the multi-term time-fractional integro-differential problem (1) has at least one mild random solution on $[0, b]$.

Proof. As in the proof of Theorem 2, we show that the operator $\mathcal{N}: D(\omega) \rightarrow D(\omega)$ has a stochastic fixed point. We know that $\mathcal{N}$ is a random operator with stochastic domain which is continuous and maps bounded sets into equicontinuous sets of $D(\omega)$. So, in order to finish the proof it is sufficient to show that $\mathcal{N}$ satisfies the Mönch's condition.

Let $\omega \in \Omega$ and $\mathbb{V}$ be a countable subset of $D(\omega)$ and $\mathbb{V} \subseteq \overline{\operatorname{con}}(\{0\} \cup \mathcal{N}(\omega)(\mathbb{V}))$. Since $\chi_{\mathcal{C}}((\mathcal{N}(\omega) \mathbb{V}))$ has a maximum, let $\left\{z^{(m)}(\cdot, \omega)\right\}_{m=1}^{\infty} \subset(\mathcal{N}(\omega) \mathbb{V})$ be a denumerable set which attain its maximum. Then there exists a set $\left\{\vartheta^{(m)}(\cdot, \omega)\right\}_{m=1}^{\infty} \subset \mathbb{V}$ such that

$$
\left(\mathcal{N}(\omega) \vartheta^{(m)}\right)(t)=z^{m}(\cdot, \omega) \text { for all } t \in[0, b] .
$$

We choose $L>0$ such that

$$
K=\frac{2 M\left(1+\zeta_{B}\right)}{\Gamma(1+\beta)} \sup _{t \in[0, b]} \int_{0}^{b}(b-s)^{\beta} h(s, \omega) e^{L(s-t)} d s<1 .
$$


Now, by (4), we derive that

$$
\begin{aligned}
& \chi\left(\left\{\left(\mathcal{N}(\omega) \vartheta^{(m)}\right)(t)\right\}_{m=1}^{\infty}\right) \\
\leq & 2 \frac{M\left(1+\zeta_{B}\right)}{\Gamma(1+\beta)} \int_{0}^{t}(t-s)^{\beta} h(s, \omega) \chi\left(\left\{\vartheta^{(m)}(s)\right\}_{m=1}^{\infty}\right) d s \\
\leq & 2 \frac{M\left(1+\zeta_{B}\right)}{\Gamma(1+\beta)} \int_{0}^{b}(b-s)^{\beta} h(s, \omega) e^{L s} \sup _{s \in[0, b]}\left[e^{-L s} \chi\left(\left\{\vartheta^{(m)}(s)\right\}_{m=1}^{\infty}\right)\right] d s \\
\leq & 2 \frac{M\left(1+\zeta_{B}\right)}{\Gamma(1+\beta)} v\left(\left\{\vartheta^{(m)}\right\}_{m=1}^{\infty}\right) \int_{0}^{b}(b-s)^{\beta} h(s, \omega) e^{L s} d s .
\end{aligned}
$$

By using (5) and (8), we get

$$
\begin{aligned}
v\left(\left\{z^{(m)}\right\}_{m=1}^{\infty}\right) & \leq \sup _{t \in[0, b]} e^{-L t} \chi\left(\left\{\left(\mathcal{N}(\omega) \vartheta^{(m)}\right)(t)\right\}_{m=1}^{\infty}\right) \\
& \leq \sup _{t \in[0, b]} e^{-L t} \frac{2 M\left(1+\zeta_{B}\right)}{\Gamma(1+\beta)} v\left(\left\{\vartheta^{(m)}\right\}_{m=1}^{\infty}\right) \int_{0}^{b}(b-s)^{\beta} h(s, \omega) e^{L s} d s \\
& \leq \frac{2 M\left(1+\zeta_{B}\right)}{\Gamma(1+\beta)} v\left(\left\{\vartheta^{(m)}\right\}_{m=1}^{\infty}\right) \sup _{t \in[0, b]} \int_{0}^{b}(b-s)^{\beta} h(s, \omega) e^{L(s-t)} d s \\
& \leq K v\left(\left\{\vartheta^{(m)}\right\}_{m=1}^{\infty}\right) .
\end{aligned}
$$

Thus, we have

$$
\begin{aligned}
& v\left(\left\{\vartheta^{(m)}(\cdot, \omega)\right\}_{m=1}^{\infty}\right)=v(\mathbb{V}) \leq v(\overline{\operatorname{con}}(\{0\} \cup \mathcal{N}(\omega)(\mathbb{V}))) \\
= & v\left(\left\{z^{(m)}(\cdot, \omega)\right\}_{m=1}^{\infty}\right) \leq K v\left(\left\{\vartheta^{(m)}(\cdot, \omega)\right\}_{m=1}^{\infty}\right) .
\end{aligned}
$$

From (7), we obtain

$$
v\left(\left\{\vartheta^{(m)}(\cdot, \omega)\right\}_{m=1}^{\infty}\right)=v(\mathbb{V})=v\left(\left\{z^{(m)}(\cdot, \omega)\right\}_{m=1}^{\infty}\right)=0 .
$$

By the definition of $v$, we get

$$
\chi\left(\left\{\vartheta^{(m)}(t, \omega)\right\}_{m=1}^{\infty}\right)=\chi\left(\left\{z^{(m)}(t, \omega)\right\}_{m=1}^{\infty}\right)=0 \text { for every } t \in[0, b] .
$$

From (H4), we obtain

$$
\begin{aligned}
& \chi\left(\left\{F\left(s, \vartheta^{(m)}(s, \omega), \int_{0}^{s} \mathcal{B}(s, \tau) \vartheta^{(m)}(\tau, \omega) d \tau, \omega\right)\right\}_{m=1}^{\infty}\right) \\
\leq & h(t, \omega)\left(1+\zeta_{B}\right) \chi\left(\left\{\vartheta^{(m)}(t, \omega)\right\}\right)=0
\end{aligned}
$$

which implies that

$$
\mathcal{S}:=\left\{F\left(s, \vartheta^{(m)}(s, \omega), \int_{0}^{s} \mathcal{B}(s, \tau) \vartheta^{(m)}(\tau, \omega) d \tau, \omega\right)\right\}_{m=1}^{\infty}
$$


is relatively compact for almost all $t \in[0, b]$ in $\mathbb{X}$. Since $\left\{\vartheta^{(m)}(\cdot, \omega)\right\}_{m=1}^{\infty} \in D(\omega)$, by (H3), we derive that $\mathcal{S}$ is uniformly integrable for a.e $t \in[0, b]$. From Definition 10, we conclude that $\mathcal{S}$ is semicompact and by Lemma 4 , the set

$$
\left\{\int_{0}^{t} \mathcal{T}_{\beta, \gamma_{k}}(t-s) F\left(s, \vartheta^{(m)}(s, \omega), \int_{0}^{s} \mathcal{B}(s, \tau) \vartheta^{(m)}(\tau, \omega) d \tau, \omega\right) d s\right\}_{m=1}^{\infty}
$$

is relatively compact in $\mathbb{X}$. Hence, by $(6),\left\{z^{(m)}(\cdot, \omega)\right\}_{m=1}^{\infty}$ is also relatively compact in $\mathcal{C}([0, b], \mathbb{X})$. Since $\chi_{\mathcal{C}}$ is a nonsingular, monotone and regular measure of noncompactness, then by Mönch condition, we have

$$
\chi_{\mathcal{C}}(\mathbb{V}) \leq \chi_{\mathcal{C}}(\overline{\overline{c o n}}(\{0\} \cup \mathcal{N}(\omega)(\mathbb{V})))=\chi_{\mathcal{C}}\left(\left\{z^{(m)}(\cdot, \omega)\right\}_{m=1}^{\infty}\right)=0,
$$

which shows that $\mathbb{V}$ is relatively compact in $\mathcal{C}([0, b], \mathbb{X})$. The proof is completed.

Remark 2. In comparison to Theorem 2, the result obtain in Theorem 3 is more general and interesting. Due to the choice of the measure of noncompactness, we can notice that inequality (2) in Theorem 2 is not necessary in Theorem 3.

In Theorem 3, when we replace the condition $(\mathbf{H} 4)$ by $\left(\mathbf{H}^{\prime}\right)$, we obtain the following result where the condition (H5) is released.

Theorem 5. Under the assumptions $(\mathrm{H1}),(\mathrm{H} 2),\left(\mathrm{H}^{\prime}\right)$ and $(\mathrm{H} 4)$, the multi-term time-fractional integro-differential problem (1) has at least one mild random solution on $[0, b]$.

Proof. As in the proof of Theorem 3, there exists a random function $r: \Omega \rightarrow \mathbb{R}-\{0\}$ such that the operator $\mathcal{N}: D(\omega) \rightarrow D(\omega)$ is a random operator with stochastic domaine $D(\omega)=$ $\{\vartheta \in \mathcal{C}([0, b], \mathbb{X}):\|\vartheta\| \leq r(\omega)\}$. Furthermore, we know that $\mathcal{N}$ is a random operator with stochastic domain which is continuous and maps bounded sets into equicontinuous sets of $D(\omega)$. So, we only need to check that $\mathcal{N}$ satisfies the Mönch's condition. Following a similar argument as in the proof of Theorem 3, one can verify the conclusion.

Remark 3. The random differential equation with delay is a special type of random functional differential equations. The random functional differential equations with state-dependent delay have many important applications in mathematical models of real phenomena. By applying the ideas and techniques as in this article and making some appropriate conditions, one can obtain the existence results for a class of multi-term time-fractional random integro-differential equations with state-dependent delay.

\section{A Nontrivial Example of Application of Theorem 2}

In this section, we give a nontrivial example to illustrate our main results.

Example 1. Let $\beta, \gamma_{k}>0 k=1,2, \cdots, m$ be such that $0<\beta \leq \gamma_{m} \leq \cdots \leq \gamma_{1} \leq 1$. We consider the following problem with random effects: 


$$
\left\{\begin{array}{l}
{ }^{C} D^{1+\beta} u(t, x, \omega)+\sum_{k=1}^{m} \alpha_{k}{ }^{C} D^{\gamma_{k}} u(t, x, \omega)=\frac{\partial^{2} u(t, x, \omega)}{\partial^{2} x} \\
\frac{(1+t)^{-1 / 3}}{20} Q(\omega)\left[\sin (u(t, x, \omega))+\int_{0}^{t} \cos (t-s) u(s, x, \omega) d s\right], x \in[0, \pi], t \in[0,1], \omega \in \Omega \\
u(t, 0, \omega)=u(t, \pi, \omega)=0, t \in[0,1], \omega \in \Omega, \\
u(0, x, \omega)=u_{0}(x, \omega), x \in[0, \pi], \omega \in \Omega, \\
\left.\frac{\partial u(t, x, \omega)}{\partial t}\right|_{t=0}=u_{1}(x, \omega), x \in[0, \pi], \omega \in \Omega,
\end{array}\right.
$$

where $Q$ is a real-valued random variable, $u_{0}, u_{1}:[0, \pi] \times \Omega \rightarrow \mathbb{R}$ are given functions and $(\Omega, \mathcal{F}, \mathrm{P})$ a complete probability space. Let $\mathbb{X}=\mathbb{L}^{2}([0, \pi], \mathbb{R})$ and define the operator $\mathcal{A}: D(\mathcal{A}) \subset \mathbb{X} \rightarrow \mathbb{X}$ by $\mathcal{A} z=z^{\prime \prime}$, where

$$
D(\mathcal{A})=\left\{z \in \mathbb{X}: z, z^{\prime} \text { are absolutely continuous, } z^{\prime \prime} \in \mathbb{X}, z(0)=z(\pi)=0\right\} .
$$

then

$$
\mathcal{A} z=\sum_{n=1}^{\infty} n^{2}\left(z, w_{n}\right) w_{n}, z \in D(\mathcal{A})
$$

where $w_{n}(\theta)=\sqrt{\frac{2}{\pi}} \sin (n \theta), n=1,2, \cdots$ is the orthogonal set of eigenvectors of $\mathcal{A}$. Thus, $\mathcal{A}$ generates a strongly continuous cosine family $\{C(t)\}_{t \in \mathbb{R}}$ given by

$$
C(t) z=\sum_{n=1}^{\infty} \cos (n t)\left(z, w_{n}\right) w_{n} \text { for } z \in \mathbb{X}
$$

Since $\beta, \gamma_{k}>0 k=1,2, \cdots, m$ be such that $0<\beta \leq \gamma_{m} \leq \cdots \leq \gamma_{1} \leq 1$, by Theorem 1, we deduce that $\mathcal{A}$ generates a bounded $\left(\beta, \gamma_{k}\right)$-resolvent family

$$
\mathcal{R}_{\beta, \gamma_{k}}(t) z=\int_{0}^{\infty} \frac{1}{t^{(1+\beta) / 2}} \Phi_{(1+\beta) / 2}\left(s t^{-(1+\beta) / 2}\right) C(s) z d s, \quad z \in \mathbb{X}, \quad t \in[0,1],
$$

where

$$
\Phi_{(1+\beta) / 2}(y)=\sum_{n=0}^{\infty} \frac{(-y)^{n}}{n ! \Gamma(-(\beta(n+1))-n)^{2}}, \quad y \in \mathbb{C},
$$

is the Wright functions. Furthermore, we define

$$
\vartheta(t, \omega)(x)=u(t, x, \omega) \text { for } t \in[0,1], \quad x \in[0, \pi] \text { and } \omega \in \Omega
$$

and

$$
\vartheta_{0}(\omega)(x)=u_{0}(x, \omega), \quad \vartheta_{1}(\omega)(x)=u_{1}(x, \omega) \text { for } x \in[0, \pi] \text { and } \omega \in \Omega .
$$

For every $t \in[0,1], x \in[0, \pi]$ and $\omega \in \Omega$, define

$$
F\left(t, \vartheta(t, \omega), \int_{0}^{t} \mathcal{B}(t, s) \vartheta(s, \omega) d s, \omega\right)(x)=f(t, \omega)\left[\sin (u(t, x, \omega))+\int_{0}^{t} \cos (t-s) u(s, x, \omega) d s\right]
$$


where $f(t, \omega)=\frac{(1+t)^{-1 / 3} Q(\omega)}{20}$. Then Equation (11) can be rewritten in the abtract form of Equation (1). From the definition of the nonlinear term $F$, we have

$$
\begin{aligned}
& \left\|F\left(t, \vartheta(t, \omega), \int_{0}^{t} \mathcal{B}(t, s) z_{1}(s, \omega) d s, \omega\right)-F\left(t, \tilde{\vartheta}(t, \omega), \int_{0}^{t} \mathcal{B}(t, s) z_{2}(s, \omega) d s, \omega\right)\right\| \\
& \leq f(t, \omega)\left[\|\vartheta(\cdot, \omega)-\tilde{\vartheta}(\cdot, \omega)\|+\zeta_{B}\left\|z_{1}(\cdot, \omega)-z_{2}(\cdot, \omega)\right\|\right],
\end{aligned}
$$

where $\zeta_{B}=\sup _{t \in[0,1]} \int_{0}^{t} \cos (t-s) d s \leq 1$. Therefore

$$
\left\|F\left(t, \vartheta(t, \omega), \int_{0}^{t} \mathcal{B}(t, s) z(s, \omega) d s, \omega\right)\right\| \leq f(t, \omega)\left[\|\vartheta(\cdot, \omega)\|+\zeta_{B}\|z(\cdot, \omega)\|\right]
$$

and for any bounded and contable set $\mathbb{V}$ of $\mathbb{X}$, we obtain

$$
\chi\left(F\left(t, \mathbb{V}(t), \int_{0}^{t} \mathcal{B}(t, s) \mathbb{V}(s) d s, \omega\right)\right) \leq h(t, \omega)\left[\chi(\mathbb{V})+\zeta_{B} \chi(\mathbb{V})\right]
$$

where $h(t, \omega)=f(t, \omega)$. Taking $p_{1}=p_{2}=\frac{1}{2}$ and $\beta=\frac{3}{4}$, we have

$$
P_{1}=P_{2}=\sqrt{\frac{2}{5}} \text { and }\|f(\cdot, \omega)\|=\frac{3}{50}\left[(2)^{5 / 6}-1\right]^{1 / 2}|Q(\omega)| \leq 0.06 \times|Q(\omega)| .
$$

If $\frac{2 M \sqrt{2 / 5}}{\Gamma(7 / 4)} 0.06 \times|Q(\omega)|<\frac{1}{2}$ then by Theorem 2, the random system (11) has at least one random mild solution.

\section{Conclusions}

Random fractional integro-differential equations are one of the most important research topics in the past thirty years. In this paper, we investigate the existence of mild solutions to a multi-term fractional integro-differential Equation (1) with random effects (see Theorems 2-5). A nontrivial example illustrating Theorem 2 is also given.

Author Contributions: Writing original draft, A.D. and W.-S.D. Both authors have read and agreed to the published version of the manuscript.

Funding: The second author is partially supported by Grant No. MOST 110-2115-M-017-001 of the Ministry of Science and Technology of the Republic of China.

Institutional Review Board Statement: Not applicable.

Informed Consent Statement: Not applicable.

Data Availability Statement: Not applicable.

Acknowledgments: The authors wish to express their hearty thanks to the anonymous referees for their valuable suggestions and comments.

Conflicts of Interest: The authors declare no conflict of interest.

\section{References}

1. Podlubny, I. Fractional Differential Equations; Academic Press: NewYork, NY, USA, 1999.

2. Kilbas, A.A.; Srivastava, H.M.; Trujillo, J.J. Theory and Applications of Fractional Differential Equations, North-Holland Mathematics Studies 204; Elsevier Science: Amsterdam, The Netherlands, 2006.

3. Keyantuo, V.; Lizama, C.; Warma, M. Asymptotic behavior of fractional order semilinear evolution equations. Differ. Integral Equ. 2013, 26, 757-780. 
4. Trong, L.V. Decay mild solutions for two-term time fractional differential equations in Banach spaces. J. Fixed Point Theory Appl. 2016, 18, 417-432

5. Singh, V.; Pandey, D.N. Controllability of multi-term time-fractional differential systems. J. Control Decis. 2020, 7, 109-125. [CrossRef]

6. Pardo, E.A.; Lizama, C. Mild solutions for multi-term time-fractional differential equations with nonlocal initial conditions. Electron. J. Differ. Equ. 2014, 39, 1-10.

7. Bharucha-Reid, A.T. Random Integral Equations; Academic Press: New York, NY, USA, 1972.

8. Tsokos, C.P.; Padgett, W.J. Random Integral Equations with Applications in Life Sciences and Engineering; Academic: New York, NY, USA, 1974.

9. Dhage, B.C.; Ntouyas, S.K. Existence and attractivity results for nonlinear first order random differential equations. Opusc. Math. 2010, 30, 411-429. [CrossRef]

10. Edsinger, R. Random Ordinary Differential Equations. Ph.D. Thesis, University of California, Berkeley, Berkeley, CA, USA, 1968.

11. Lungan, C.; Lupulescu, V. Random differential equations on time scales. Electron. J. Differ. Equ. 2012, 86, 1-14.

12. Yang, D.; Wang, J. Non-instantaneous impulsive fractional- order implicit differential equations with random effects. Stoch. Anal. Appl. 2017, 35, 719-741. [CrossRef]

13. Abbas, S.; Benchohra, M.; Henderson, J. Random Caputo-Fabrizio fractional differential inclusions. Math. Model. Control 2021, 1, 102-111. [CrossRef]

14. Singh, V.; Pandey, D.N. Mild Solutions for Multi-Term Time-Fractional Impulsive Differential Systems. Nonlinear Dyn. Syst. Theory 2018, 18, 307-318.

15. Chang, Y.K.; Ponce, R.V. Mild solutions for a multi-term fractional differential equation via resolvent operators. AIMS Math. 2021, 6, 2398-2417. [CrossRef]

16. Engl, H.W. A general stochastic fixed-point theorem for continuous random operators on stochastic domains. J. Math. Anal. Appl. 1978, 66, 220-231. [CrossRef]

17. Banas̀, J.; Goebel, K. Measures of Noncompactness in Banach Spaces; Lect. Notes Pure Appl. Math. 60; Marcel Dekker: New York, NY, USA, 1980.

18. Deimling, K. Nonlinear Functional Analysis; Springer: New York, NY, USA, 1985.

19. O'Regan, D.; Precup, R. Existence criteria for integral equations in Banach spaces. J. Inequalities Appl. 2001, 6, 77-97. [CrossRef]

20. Kamenskii, M.; Obukhovskii, V.; Zecca, P. Condensing Multivalued Maps and Semilinear Differential Inclusions in Banach Spaces; De Gruyter: Berlin, Germany, 2001.

21. Mönch, H. Boundary value problems for nonlinear ordinary differential equations of second order in Banach spaces. Nonlinear Anal. 1980, 4, 985-999. [CrossRef] 